\title{
Strain-induced domain formation in two-dimensional colloidal systems
}

\author{
S. Bleil ${ }^{1}$, H. H. von Grünberg ${ }^{2}$, J. Dobnikar ${ }^{2,3}$, \\ R. Castañeda-Priego ${ }^{4}$ and C. Bechinger ${ }^{1}$ \\ 1 2. Physikalisches Institut, Universität Stuttgart - 70550 Stuttgart, Germany \\ 2 Institut für Chemie, Karl-Franzens-Universität - 8010 Graz, Austria \\ 3 Institute Josef Stefan - Jamova 39, SI-1000 Ljubljana, Slovenia \\ 4 Instituto de Fisica, Universidad de Guanajuato - 37150 Leon, Mexico
}

\author{
PACS. 82.70.Dd - Colloids. \\ PACS. 68.43.Hn - Structure of assemblies of adsorbates (two- and three-dimensional cluster- \\ ing). \\ PACS. 64.70.Rh - Commensurate-incommensurate transitions.
}

\begin{abstract}
We report on the spontaneous formation of domains in colloidal monolayers subjected to a triangular substrate, the latter created by an optical interference pattern. Upon variation of the particle number density we observe a transition from a homogeneous phase to a network of pronounced domain structures. We demonstrate that this transition is driven by the elastic strain between the colloidal system and the underlying substrate.
\end{abstract}

Thin layers adsorbed on crystalline surfaces are generally strained owing to their lattice mismatch with respect to the substrate. When the periodicity of the adsorbate is a multiple of the lattice constant of the substrate, a commensurate structure will form which is typically encountered for chemisorbed films with large adsorbate-substrate interactions. In contrast, if the mutual interactions between the adsorbate atoms dominate, as is the case in physisorbed layers, slight lattice mismatches will cause incommensurate structures. It has been realized that during the growth of slightly incommensurate layers self-organized quantumdots or quantum-wires can be obtained under appropriate conditions. Such structures are of enormous physical and technological interest due to their intriguing optical, electronic and magnetic properties $[1-3]$.

Qualitative aspects of commensurate-incommensurate transitions are already described in the classical problem of the Frenkel-Kontorova chain (see, e.g., [4]). Beyond a certain mismatch between the atoms and the periodic substrate potential, large regions ("domains") with nearly commensurate ordering develop which are separated by rather narrow domain walls. The $2 \mathrm{D}$ generalization of this problem was first theoretically studied by Shiba [5]; it is based on a Hamiltonian like that in eq. (2), consisting of two terms which arise from the substrate-adatom and the adatom-adatom interaction. For monolayers on structured surfaces, the competing effect of these two interactions can lead to a rich variety of different adsorbate phases and phase transitions (domain-wall theories are reviewed in $[4,6]$ ).

In this letter we study colloidal monolayers on triangular substrates. With increasing particle number density, pronounced domain formation is found with clusters of characteristic sizes 
and shapes. At certain densities we observe domain structures consisting of commensurate domains separated by a honeycomb array of domain walls. Similar hexagonal expanded structures with super-light [4] domain walls have been theoretically predicted for Xe sub-monolayers on graphite [7] and subsequently also experimentally observed in synchrotron X-ray [8] and scanning tunneling microscopy (STM) experiments [9].

It has already been demonstrated that colloids exposed to strong light fields can serve as model systems for atomic monolayers [10,11]. Due to their size, colloids can be directly observed under the microscope and conveniently tracked over many hours by appropriate video-microscopy techniques [12]. Using these trajectories to compute internal energies, we have been able to show by means of a quantitative analysis that domains are the energetically most favorable structure because they allow the system to effectively release elastic stress.

A similar quantitative analysis of real-space data in atomic systems (as, for example, those of the STM experiment in [9]) is currently not possible. Besides this there are other advantages of colloidal over atomic systems: i) In atomic systems one cannot easily distinguish between pure strain effects and system specific interactions. ii) The lattice mismatch can not be varied independently but typically depends on the atomic composition of the substrate whereas for colloids on light-induced substrates all relevant parameters for domain formation - such as substrate strength, lattice type, lattice constant, pair-interaction - can be varied independently and over large ranges. iii) Other than in our system, the substrate in atomic systems need not be a perfect lattice, but can suffer itself from elastic deformations or defects.

The experiments were performed with aqueous suspensions of highly charged polystyrene (PS) spheres with radius $R=1.4 \mu \mathrm{m}$ which interact via a Yukawa pair-potential

$$
u(r) / k_{B} T=\left(\frac{Z_{\text {eff }} \exp [\kappa R]}{1+\kappa R}\right)^{2} \lambda_{B} \frac{\exp [-\kappa r]}{r}
$$

with $r$ the particle distance, $\kappa^{-1}$ the Debye screening length, $Z_{\text {eff }}$ the effective colloidal charge and $\lambda_{B}$ the Bjerrum length $(0.72 \mathrm{~nm}$ in water). As sample cell we used a cuvette with $200 \mu \mathrm{m}$ spacing which was connected to a closed deionization circuit where we obtain screening lengths up to approx. $550 \mathrm{~nm}$ [11]. Substrate potentials with lattice spacing $a_{L}$ were created by overlapping three linearly polarized beams of a $\mathrm{Nd}: \mathrm{YVO}_{4}$ laser which are directed from above into the sample cell. The interference pattern acts as a substrate with substrate strength $V_{g}$, whose value is obtained from analyzing the particle distribution across the substrate at highly diluted conditions where pair interactions can be safely ignored. The vertical component of the light pressure pushes the particles towards the equally charged bottom plate of the cuvette and confines the system effectively to $2 \mathrm{D}$. With another scanned optical laser tweezers $(\lambda=$ $488 \mathrm{~nm}$ ) we created a lateral boundary box for the $2 \mathrm{D}$ colloidal system to adjust the particle number density with an accuracy well below $1 \%$. For details we refer to previous papers [1113]. In the absence of the light pattern and at the particle number densities considered here, the colloids form a fluid with the mean particle distance $d_{n n}$ as the characteristic length scale. From this we define the filling factor $\eta=\left(a_{L} / d_{n n}\right)^{2}$ as the ratio of the unit cell areas of the substrate lattice $\left(\sqrt{3} a_{L}^{2} / 2\right)$ and that of the substrate-free colloidal system $\left(\sqrt{3} d_{n n}^{2} / 2\right)$.

Figure 1 shows typical configurations of colloids on a light-induced (111) surface $\left(a_{L}=\right.$ $5.5 \mu \mathrm{m}$ and $V_{g}=6.9 k_{B} T$ ) for different $\eta$. Initially $V_{g}$ was chosen to be sufficiently small in order to allow particle fluctuations across the potential barriers, i.e., to reach thermal equilibrium. Then, $V_{g}$ was gradually increased until all particles became eventually trapped at their lattice sites. This procedure corresponds to cooling the substrate and is here - in contrast to atomic systems where the adsorbat is often quenched onto a cold surface- performed in a wellcontrolled manner. While we observe small differences in the particle configurations when 


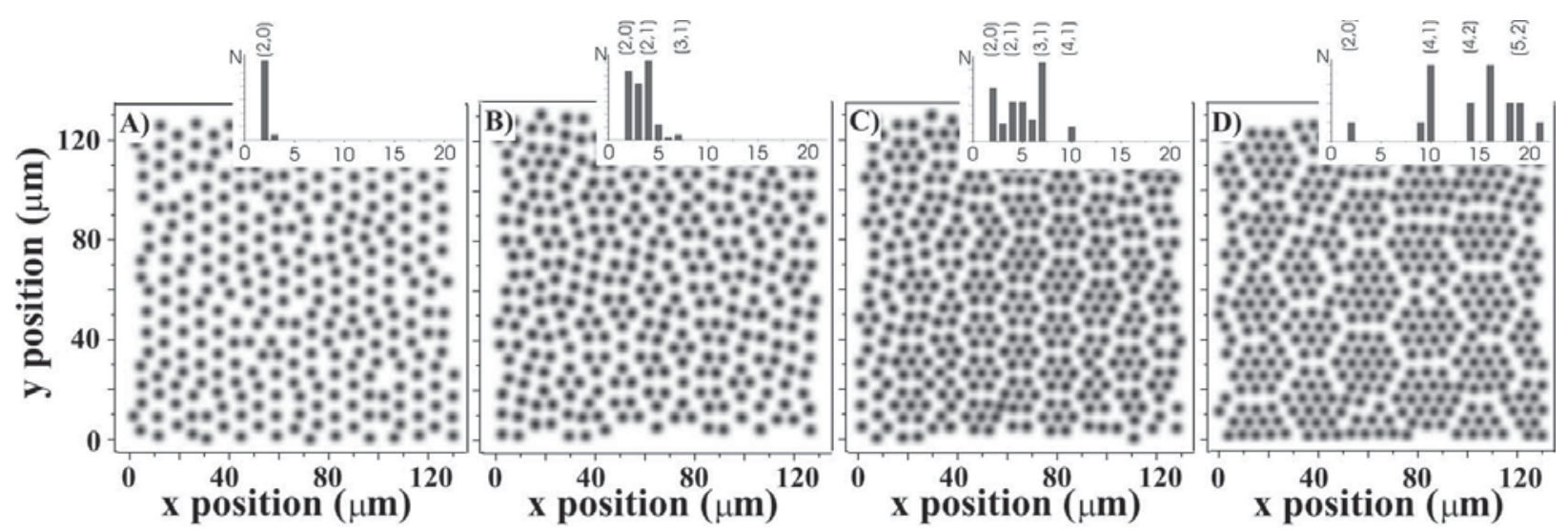

Fig. 1 - Colloidal particles in a hexagonal light field with $V_{g}=6.9 k_{B} T$ for $\eta=0.34$ (A), 0.48 (B), 0.59 (C) and 0.69 (D). To enhance the visibility of the domain walls, the snapshots are artificially defocused. Histograms in the inset figures quantify the relative occurrence of particle numbers belonging to a single domain.

repeating the experiment, the results presented in the following are independent of the details of how $V_{g}$ was increased. For $\eta=0.34$ the colloids form over large areas hexagonal structures which have a lattice constant $\sqrt{3} a_{L}$ and are rotated by $30^{\circ}$ with respect to the substrate lattice (fig. 1A). This structure corresponds to the $\sqrt{3} R 30^{\circ}$ triangular commensurate lattice predicted for $\eta=1 / 3$ and is schematically shown in fig. 2a. Increasing $\eta$ to $0.48,0.59$ and 0.69 we observe the formation of domain structures, i.e. regularly formed particle clusters which become particularly pronounced at higher $\eta$ (figs. 1B, C, D). In the following, a domain is defined as a cluster of particles, placed on next-neighbor lattice sites, with two of these clusters being separated from each other by domain walls, i.e. single rows of unoccupied sites. Although the particle interaction is short-ranged, the domain walls form a rather regular network with the average domain size increasing with growing $\eta$. Such a systematic increase of the domain size with $\eta$ was not found for atomic systems, yet [9]. To quantify the domain sizes in fig. 1, we plot as insets histograms of the particle numbers comprising the clusters (averaged over several experimental runs). It is obvious that this domain formation cannot be driven by the (repulsive!) electrostatic particle interaction because this would favor rather uniform particle distances compared to the observed clusters (with relatively large particle interactions) and empty domain walls in between.

To understand the origin of domain formation we calculate the energy $U$ of the system,

$$
U=U_{Y}+U_{S}=\sum_{i<j} u\left(r_{i j}\right)+V_{g} \sum_{i} f\left(x_{i}, y_{i}\right)
$$

with $\left(x_{i}, y_{i}\right)$ being the particle positions. The first term takes account of the pair interaction, $U_{Y}$, with the Yukawa pair-potential from eq. (1), while the second term $\left(U_{S}\right)$ describes the particle interaction with the hexagonal substrate

$$
f(x, y)=-\frac{2}{9}\left(\frac{3}{2}+\cos \frac{4 \pi y}{\sqrt{3} a_{L}}+2 \cos \frac{2 \pi y}{\sqrt{3} a_{L}} \cos \frac{2 \pi x}{a_{L}}\right) .
$$

As experimental parameters for the Debye screening length and the effective charge we determined $\kappa^{-1}=580 \pm 60 \mathrm{~nm}$ and $Z_{\text {eff }} \approx 5400$ in a way explained further below. With the experimentally determined particle positions we can now calculate the energy per particle 

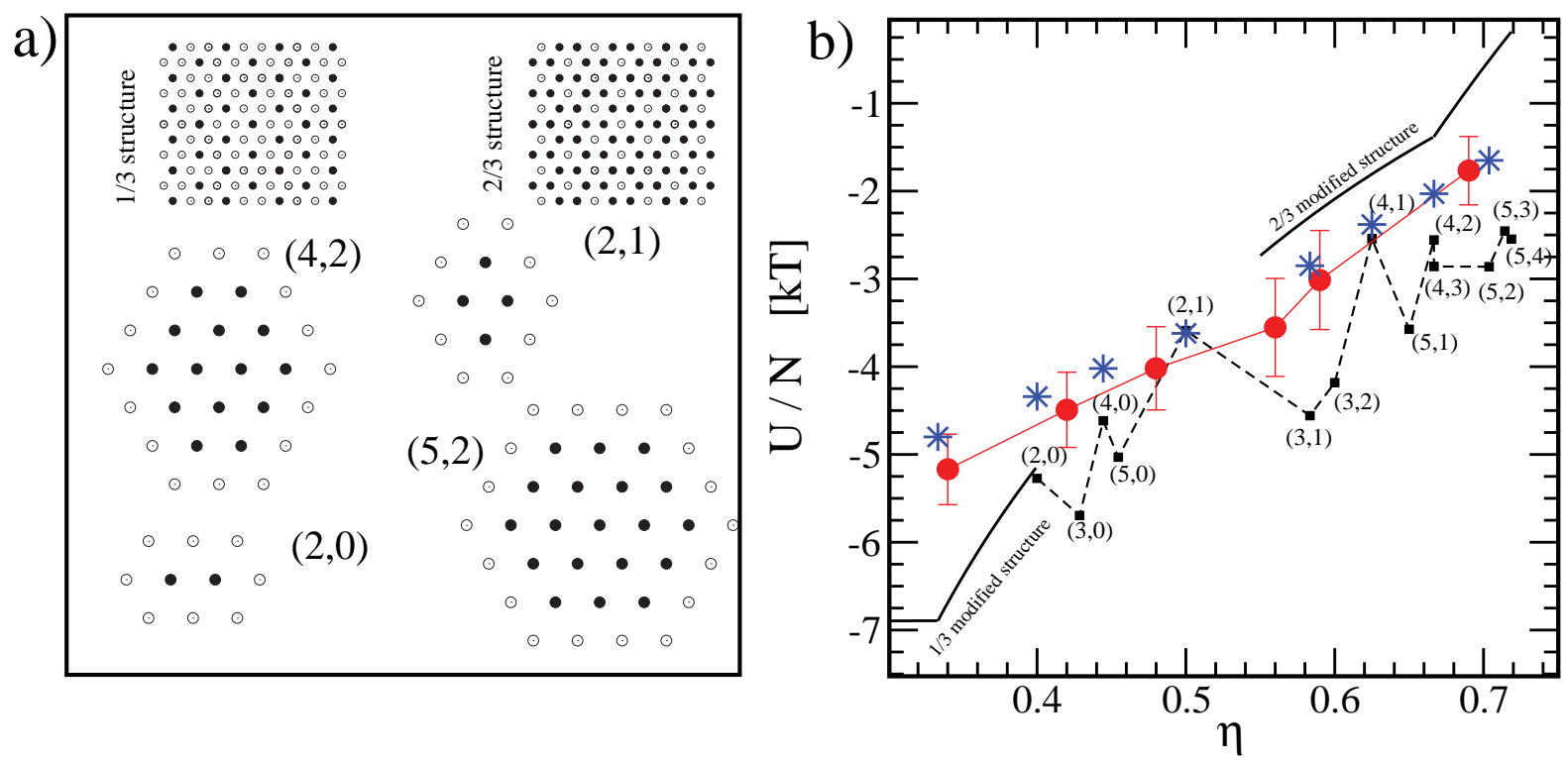

Fig. 2 - a) Possible structures of particles (solid circles) adsorbed onto a triangular substrate (open circles) for different $\eta$. The notation for the cluster structures $\left(n n, n_{j}\right)$ is explained in the text. b) Energy per colloid vs. $\eta$ for $V_{g}=6.9 k_{B} T$ : Taken from experiment (closed circles), obtained from MD simulations (stars) and theoretically calculated for various area-filling, relaxed domain structures (filled squares with dashed lines as guide to the eye).

$U / N$ from single snapshots similar to those shown in fig. 1 . The solid circles in fig. $2 \mathrm{~b}$ show the results for different $\eta$ after averaging over several hundred snapshots each. The error bars correspond to the uncertainty in our experimental parameters. The results are in excellent agreement with the results of Monte Carlo (MC) simulations which were performed according to the experimental conditions (star symbols in fig. $2 \mathrm{~b}$ ). For the simulations we considered a finite-size system of about 1000 particles with a rigid boundary realized by immobile colloids, and a switching-on procedure for $V_{g}$ as realized in the experiment.

More information about domain formation is gained by analyzing those particle configurations which minimize eq. (2). For the sake of simplicity, we considered only structures most frequently observed in simulations and experiments, namely symmetrically shaped clusters. They are characterized by $2 n_{j}+1$ rows, $n n$ particles in the central row, $(n n-1)$ particles in the adjacent rows and so forth (for examples see fig. 2a). In the following these clusters are referred to as $\left(n n, n_{j}\right)$ clusters. Together with the empty sites surrounding them, these clusters form characteristic domains which allow the substrate to be completely covered with filling factor

$$
\eta=\frac{2 n n\left(1+2 n_{j}\right)-2 n_{j}\left(n_{j}+1\right)}{\left(n_{j}+1\right)\left(4(n n+2)-4-2\left(n_{j}+1\right)\right)} .
$$

For a few of these domain types we numerically determined relaxed ground-state structures, i.e., configurations in which each particle experiences a vanishing net force, applying periodic boundary conditions adapted to the respective domain-type symmetry. The corresponding energies, plotted in fig. $2 \mathrm{~b}$ as filled squares, indeed capture the salient feature of the corresponding experimental curve.

Figure $2 \mathrm{~b}$ also makes a prediction regarding the most probable cluster type at a given $\eta$ : The smallest possible cluster is the $(2,0)$ cluster, forming an area-filling structure at $\eta=0.4$. This is consistent with our experimental observation that at $\eta=0.34$, if at all, only $(2,0)$ 
clusters are found (the $\left(n n, n_{j}\right)$ notation is added to the respective bars in the histograms of fig. 1). At $\eta=0.48$ and $\eta=0.59$ the most probable clusters are predicted to be $(2,1)$ and $(3,1)$ which is again consistent with our experimental findings at those $\eta$. At even higher $\eta$ the correspondence is less obvious; however, fig. 1D supports the trend as predicted from our simulation data in fig. $2 \mathrm{~b}$ of growing cluster sizes with increasing $\eta$. Starting from the $\sqrt{3} R 30^{\circ}$ commensurate phase at $\eta=1 / 3$, one can also construct other phases at $\eta>1 / 3$ and $\eta<1 / 3$ by adding or removing colloids to unoccupied and occupied sites, respectively. If the neighboring particles to these added and removed colloids are allowed to adjust within their substrate potential wells to relax their elastic stress, the energy per particle corresponds to the solid line labeled " $1 / 3$ modified structure" in fig. $2 \mathrm{~b}$. The solid line labeled " $2 / 3$ modified structure" in fig. $2 \mathrm{~b}$ shows the corresponding results for values of $\eta \approx 2 / 3$. The energy of the $\sqrt{3} R 30^{\circ}$ structure is below the experimental data point at $\eta=0.34$ and indeed fig. $1 \mathrm{~A}$ is essentially a mixture of the $\sqrt{3} R 30^{\circ}$ structure and a few $(2,0)$ clusters. In contrast, the $2 / 3$ structure, or its modified form at $\eta>2 / 3$ or $\eta<2 / 3$, with its much higher energy was never observed in our experiments.

To understand why domain formation becomes energetically more favorable compared to a more uniform surface coverage, we exemplarily discuss the situation for $\eta=2 / 3$. Here the homogeneous $2 / 3$ structure competes with $(4,2)$ and $(4,3)$ domain phases which can be realized at the same filling factor (see fig. $2 \mathrm{~b}$ ). Without relaxation effects and only considering nextneighbor interactions (which is valid for the short-ranged particle interaction considered here) the homogeneous $2 / 3$ structure has the smallest energy per particle, i.e. $U_{Y} / N=1.5 u\left(a_{L}\right)$, compared to 29 bonds per 14 particles $=29 / 14 u\left(a_{L}\right)\left(=2.07 u\left(a_{L}\right)\right)$ and $33 / 16 u\left(a_{L}\right)(=2.06$ $\left.u\left(a_{L}\right)\right)$ for the $(4,2)$ and $(4,3)$ domain phases, respectively. However, while due to symmetry the particles in the $2 / 3$ structure are fixed to the centers of their lattice sites, the empty sites constituting the domain walls allow the clusters in the $(4,2)$ and $(4,3)$ domains to expand and thus to reduce the strain such that the domain structures eventually become energetically more favorable than the homogeneous phase (see fig. 2b). This clearly demonstrates that the domain structures are favored over the homogeneous phase because of their efficient way to release the elastic stress within the clusters.

We next wish to quantify the extent of stress release, again for a configuration at $\eta=0.69$ $\left(V_{g}=24 k_{B} T\right)$. At this $\eta$ the $(5,2)$ domain type predominantly fills the surface. A schematic picture of a relaxed $(5,2)$ domain is shown in fig. 3a. The particles (black bullets) are radially displaced relative to the substrate (open circles). Owing to the symmetry the entire relaxed structure depends only on two parameters, which are the radial displacements of the first and the second shell of particles with respect to the underlying lattice sites, $\Delta r_{1}$ and $\Delta r_{2}$. Note, in particular, that the second-shell displacement in the $(1,1)$ direction does not require a third variable, since it can be expressed in terms of $\Delta r_{2}$. Because $\Delta r_{1}$ is found to be very small, it will be disregarded in the following. Assuming the surface to be entirely covered with relaxed $(5,2)$ domains, one can compute distance histograms that solely depend on $\Delta r_{2}$ for given $V_{g}$. In other words, there is a one-to-one correspondence between $\Delta r_{2}$ and $V_{g}$. The solid line in fig. $3 \mathrm{~b}$ corresponds to the result obtained from a MD simulation at $T=0$ for $\Delta r_{2}=0.1 a_{L}$ $\left(V_{g}=24 k_{B} T\right)$ and agrees very well with the experimental data [14]. To demonstrate how sensitively the calculated histograms depend on $\Delta r_{2}$ we also show the corresponding results for $\Delta r_{2}=0.15 a_{L}$ (dashed line). To visualize the extent of particle displacement from the substrate lattice sites, we plotted for comparison (dashed vertical lines) the distances appearing in a perfect triangular lattice. The good agreement found here for the distance histogram is the more remarkable because the experimentally observed domain structure at $\eta=0.69$ (fig. 1D) contains several defects and is far from being perfect.

Analyzing in this way experimentally obtained histograms for other substrate potentials 
a)
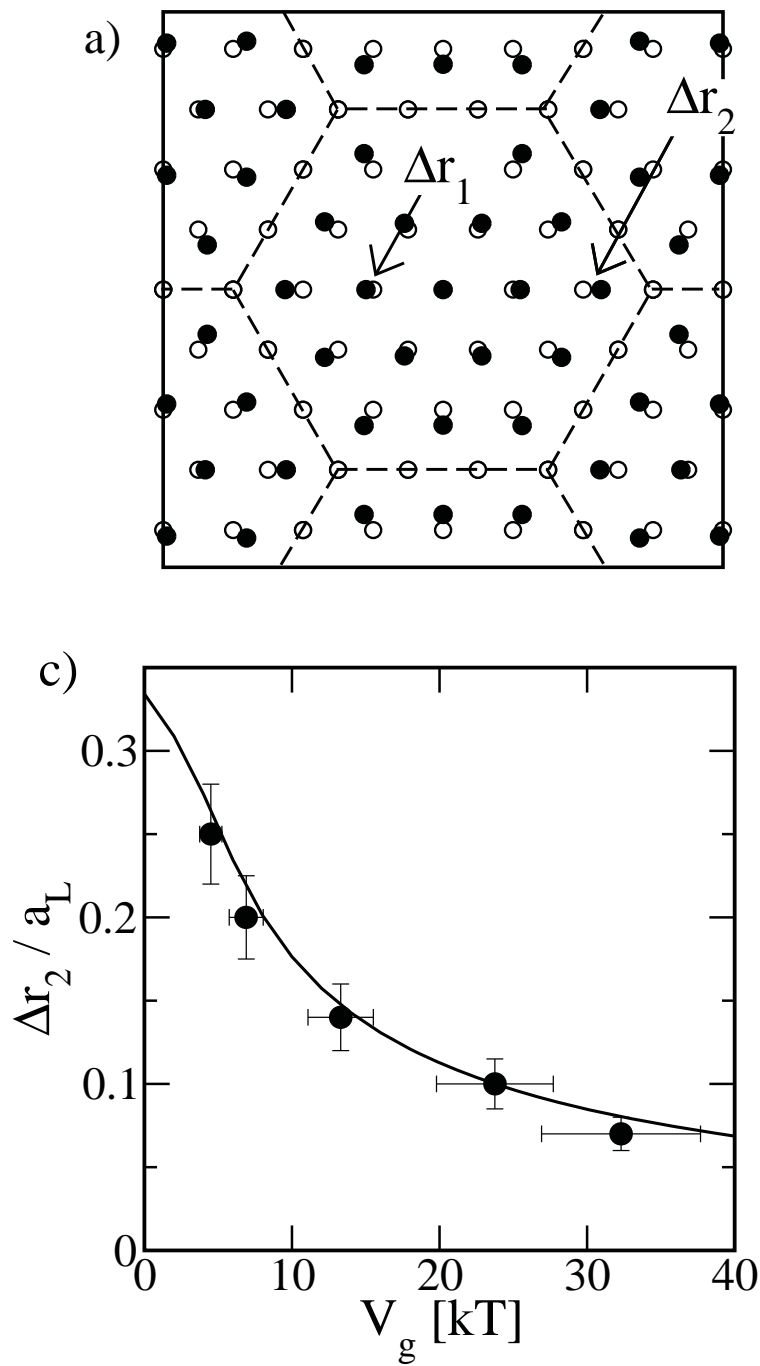

b)
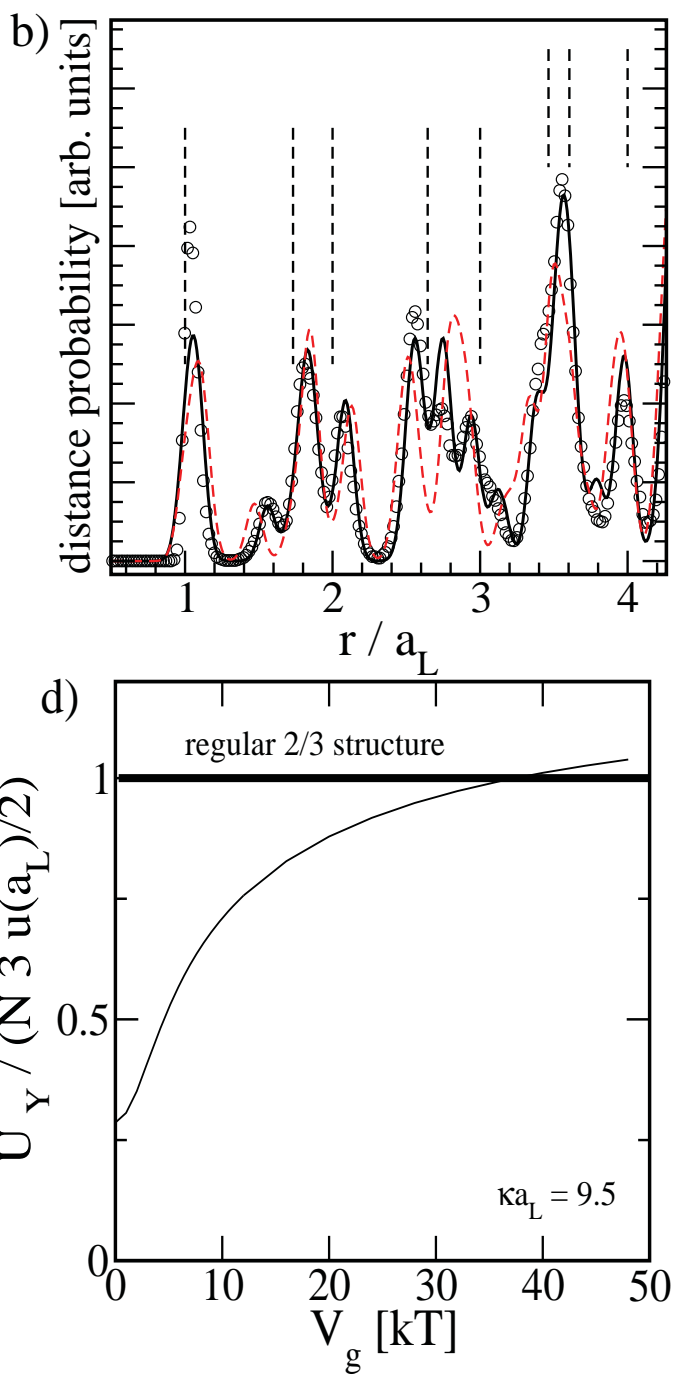

Fig. 3 - a) Picture of the relaxed $(5,2)$ domain structure, and definition of the displacements $\Delta r_{1}$ and $\Delta r_{2}$. Particles and lattice sites correspond to closed and open symbols. The dashed lines symbolize the domain walls. b) Comparison of site distances in a hexagonal lattice (vertical lines) with particle distances obtained from experiment (open circles) and numerical calculations (solid and dashed line). c) Theoretically calculated (solid line) and experimentally obtained (circles) displacements $\Delta r_{2}$ as a function of the substrate potential. d) Total pair interaction energy $U_{Y}$ per particle as a function of the substrate potential for $\eta=0.69$. At very high substrate strength the $\eta=2 / 3$ structure (thick line) becomes energetically more favorable than the domain structure (thin line).

$\left(4.5 k_{B} T<V_{g}<32 k_{B} T\right)$ we arrive at a plot that quantifies the stress relaxation in terms of $\Delta r_{2}$ vs. $V_{g}$, shown as filled circles in fig. 3c. It is compared to our ground-state calculations based on eq. (2), where $Z_{\text {eff }}$ and $\kappa^{-1}$ has been treated as adjustable parameters. Best agreement is found for the above-mentioned values which are also consistent with independent results obtained by inversion of the pair-correlation function [13]. The plot demonstrates that one can come to a quantitative understanding of the strain-induced particle displacements in these domain structures.

Figure 3d compares the total pair interaction $U_{Y} / N$ as defined in eq. (2) for $\eta=0.69$ as a function of the substrate strength. Below $V_{g} \approx 40 k_{B} T$, cluster formation (thin solid line) is energetically more favorable than the regular (i.e. unrelaxed) $2 / 3$ structure (thick line). 
For higher substrate strengths, however, the two lines cross and domain formation becomes energetically more costly. The reason for this crossover is that with increasing $V_{g}$ particle displacements with respect to the minima of the substrate potential wells require an increasing amount of energy. Accordingly, relaxation effects become less pronounced and eventually the regular $2 / 3$ structure with a uniform particle distance is favored. While this crossover is beyond the maximum substrate strengths achievable in our setup, the behavior observed in fig. $3 \mathrm{~d}$ is in agreement with results of Reichhardt et al., where the $2 / 3$ structure was predicted to be the ground state for $\eta=2 / 3$ in the limit of infinite substrate strength [15]. This clearly demonstrates that it is not only the lattice mismatch between the adsorbate and the substrate but also the substrate strength which eventually determines the resulting morphology of the adsorbed phase.

By means of additionally introduced scanned optical tweezers one can also investigate how domain formation is affected by the presence of randomly distributed pinning sites. Since domain formation is one of the most important mechanism for self-assembled formation of periodic nanostructures, it will be interesting to study how defects — as typically present in atomic systems - affect the long-range order of strain-induced domains.

$$
* * *
$$

This work was supported by a Marie Curie fellowship (MEIF-CT-2003-501789), the Transregio SFB 6018 and the Deutsche Forschungsgemeinschaft (BE 1788/3).

\section{REFERENCES}

[1] Kagan C. R., Murray C. B., Nirmal M. and Bawendi M. G., Phys. Rev. Lett., 76 (1996) 1517.

[2] Tarucha S., Austing D. G., Honda T., V. D. Hage R. J. and Kouwenhoven L. P., Phys. Rev. Lett., 77 (1996) 3613.

[3] Sun S. and Murray C. B., J. Appl. Phys., 85 (1999) 4325.

[4] Griffiths R. B., Fundamental Problems in Statistical Mechanics VII (Elsevier, Amsterdam) 1990; Bruch L. W., Cole M. W. and Zaremba E., Physical Adsorption: Forces and Phenomena (Clarendon Press, Oxford) 1997.

[5] Shiba H., J. Phys. Soc. Jpn., 46 (1979) 1852; 48 (1980) 211.

[6] Patrykeijew A., Sokolowski S. and Binder K., Surf. Sci. Rep., 37 (2000) 207.

[7] Joos B., Bergersen B. and Klein M. L., Phys. Rev. B, 28 (1983) 7219.

[8] Hong H., Peters C. J., Mak A., Birgeneau R. J., Horn P. M. and Suematsu H., Phys. Rev. B, 40 (1989) 4797; Birgeneau R. J. and Horn P. M., Science, 232 (1986) 329.

[9] Grimm B., Hövel H., Pollmann M. and Reihl B., Phys. Rev. Lett., 83 (1999) 991.

[10] Brunner M. and Bechinger C., Phys. Rev. Lett., 88; Baumgartl J., Brunner M. and Bechinger C., Phys. Rev. Lett., 93 (2004) 168301.

[11] Wei Q.-H., Bechinger C., Rudhardt D. and Leiderer P., Phys. Rev. Lett., 81 (1998) 2606.

[12] Baumgartl J. and Bechinger C., Europhys. Lett., 71 (2005) 487.

[13] Brunner M., Bechinger C., Strepp W., Lobaskin V. and von Grünberg H. H., Europhys. Lett., 58 (2002) 926.

[14] The theoretical histograms in fig. 3 are computed on the basis of relaxed $(5,2)$ structures and are transformed from bar-codes into smooth curves by folding the sum of $\delta$-functions with an appropriate Gaussian. Since at $V_{g}=24 k_{B} T$ particles cannot change optical traps, this is a good approximation as the distribution must result solely from the thermal motion of the particles about their respective $T=0$ sites.

[15] Reichhardt C. and Gronbech-Jensen N., Phys. Rev. B, 63 (2001) 054510. 